\title{
"Yo quiero estudiar por mi comunidad" Trayectorias educativas de maestras tobas/qom y mbyá guaraní en Argentina
}

Ana Carolina Hecht," Noelia Enriz, ** Mariana García Palacios, *** Soledad Aliata, ***** y Alfonsina Cantore $* * * * * *$

Recibido octubre de 2017

Aceptado marzo de 2018

\section{Resumen}

Dentro de los estudios sobre Educación Intercultural Bilingüe, las experiencias formativas de los maestros indígenas se presentan como un campo de vacancia, más aún si se tiene en foco a las docentes mujeres. Este artículo presenta dos grandes desafíos. El primero apunta a vincular las experiencias formativas de maestras tobas/qom y mbyá guaraní de dos provincias (Chaco y Misiones) con diferentes recorridos en torno a la institucionalización escolar. El segundo es de orden teórico-metodológico y refiere a la incorporación, dentro del enfoque etnográfico, de relatos de vida. Se sistematizarán tópicos como: los cambios generacionales, las relaciones de género, los saberes culturales y la escuela, las experiencias formativas en espacios religiosos, sus competencias lingüísticas, los procesos de identificación, la formación y el rol docente.

\footnotetext{
* Doctora en Antropología (UBA). Investigadora Adjunta CONICET - INAPL y docente de la Facultad de Filosofía y Letras (UBA). Investigadora del Programa de Antropología y Educación (Sección de Antropología Social, Instituto de Ciencias Antropológicas, Facultad de Filosofía y Letras, UBA). Buenos Aires, Argentina. Correo electrónico: anacarolinahecht@yahoo.com.ar

** Doctora en Antropología (UBA). Investigadora Adjunta CONICET - CESIA (IDAES-UNSAM) y docente de la Facultad de Filosofía y Letras (UBA). Investigadora del Programa de Antropología y Educación (Sección de Antropología Social, Instituto de Ciencias Antropológicas, Facultad de Filosofía y Letras, UBA). Buenos Aires, Argentina. Correo electrónico: nenriz@yahoo.com.ar

*** Doctora en Antropología (UBA). Investigadora Asistente CONICET - SEANSO (ICA-UBA) y docente de la Facultad de Filosofía y Letras (UBA). Investigadora del Programa de Antropología y Educación (Sección de Antropología Social, Instituto de Ciencias Antropológicas, Facultad de Filosofía y Letras, UBA). Buenos Aires, Argentina. Correo electrónico: mariana.garciapalacios@gmail.com

**** Licenciada en Antropología. Becaria doctoral CONICET - INAPL. Investigadora tesista del Programa de Antropología y Educación (Sección de Antropología Social, Instituto de Ciencias Antropológicas, Facultad de Filosofía y Letras, UBA). Buenos Aires, Argentina. Correo electrónico: soledadaliata@hotmail.com

***** Licenciada en Antropología. Becaria doctoral ICA/UBA. Investigadora becaria del Programa de Antropología y Educación (Sección de Antropología Social, Instituto de Ciencias Antropológicas, Facultad de Filosofía y Letras, UBA). Buenos Aires, Argentina. Correo electrónico: alfonsinacantore@gmail.com
}

\section{Palabras clave}

Mujeres indígenas; Docentes;

Educación Intercultural Bilingüe; Trayectorias educativas; Mbyá y toba/qom 


\section{"I want to study for my community". Educational Trajectories of Toba/Qom and Mbyá Guaraní Women Teachers in Argentina}

\begin{abstract}
Palavras-chave
Mulheres indígenas; Professoras:

Educação Intercultural Bilingüe; Trajetórias educacionais; Mbyá e Tobas/Qom

Abstract

Within Intercultural Bilingual Education studies, formative experiences of indigenous teachers have been little explored, even more when considering women teachers. This article addresses two big challenges, as we first attempt to relate the formative experiences of Toba/Qom and Mbyá Guaraní women teachers from two provinces (Chaco and Misiones) with different trajectories in their schooling. The second challenge is theoretical and methodological, as we aim to introduce life histories within an ethnographic approach. We systematize topics such as: intergenerational changes, gender relationships, cultural knowledge and school, religious formative experiences, linguistic capacities, identification processes, teacher training and role.

\section{"Eu quero estudar pela minha comunidade". Trajetórias Educacionais de professoras tobas/qom e mbyá guaraní na Argentina}

\section{Resumo}

Dentro das pesquisas sobre Educação Intercultural Bilíngue, as experiências formativas dos professores indígenas são um campo pouco explorado, especialmente quando o foco são professoras mulheres. Este artigo apresenta dois grandes desafios: no primeiro, pretendemos vincular as experiências formativas das professoras mulheres tobas/qom e mbyá guarani com diferentes trajetórias em torno da institucionalização escolar em duas províncias argentinas (Chaco e Misiones). O segundo desafio é de ordem teóricometodológica e refere-se à incorporação da abordagem etnográfica das histórias de vida dessas mulheres. Serão sistematizados tópicos como: mudanças geracionais, relações de gênero, conhecimento cultural e escolar, experiências formativas em espaços religiosos, competências linguísticas, processos de identificação e a formação e o papel docente.
\end{abstract}

\section{Introducción}

El interés antropológico por los procesos de escolarización en contextos interculturales en América Latina se ha ido consolidando desde principios de la década de 1980 y ha tomado como referentes a los pueblos indígenas de la región. Los tópicos abordados por la antropología de la educación incluyen aspectos amplios, como los relacionados con la enseñanza bilingüe, el aprendizaje, el currículum intercultural, la definición de la interculturalidad, la formación y capacitación para docentes indígenas, las disputas en torno de la niñez indígena en la institución escolar, entre otros (López y Küper, 2000; Rebolledo, 2009; Hecht, Enriz y García Palacios, 2016). No obstante, ha sido poco abordado el recorrido por diferentes experiencias formativas de las docentes indígenas que actualmente trabajan en el marco de la Educación Intercultural Bilingüe (en adelante, EIB). Si bien la permeabilidad de las escuelas a la diversidad étnica y lingüística es una problemática que ha sido estudiada desde distintas producciones del campo de la antropología educativa y lingüística en nuestro país (Neufeld y Thisted, 1999; Novaro y Padawer, 2013), aún se trata de un asunto que, dada su complejidad de aristas y agentes, merece ser profundizado. Por eso, la investigación expuesta en este artículo intenta contribuir con un nuevo énfasis al respecto. 
Desde hace más de una década, desarrollamos nuestras investigaciones sobre experiencias formativas escolares y no escolares (religiosas, comunitarias, etc.) con poblaciones que se autoidentifican como pertenecientes al pueblo toba/qom (Chaco) y mbyá guaraní (Misiones). ${ }^{1}$ En este artículo, se sistematizan las experiencias formativas de tres mujeres tobas/qom y mbyá guaraní que se desempeñan como maestras y auxiliares docentes, a partir de analizar entrevistas en profundidad realizadas en nuestros trabajos de campo etnográfico.

En estudios anteriores, indagamos sobre distintas aristas que hacen a la complejidad de nuestro objetivo puntual en este escrito. En Enriz y García Palacios (2008) se revisaron cuestiones relativas a la particularidad de las relaciones de género que atraviesan las mujeres mbyá-guaraní; temática luego profundizada por Cantore (2014). En García Palacios y otros (2016) también se amplió el análisis de las problemáticas del género desde un abordaje comparativo sobre referentes mujeres indígenas qom y mbyá. En relación con las mujeres tobas/qom, en García Palacios y Hecht (2017) se documentaron historias de vida de referentes del campo político y educativo, mientras que Hecht (2015) y Aliata (2017) se han centrado en trayectorias escolares de maestras y maestros tobas del Chaco. Aquí, basándonos centralmente en la sistematización de entrevistas y registros de campo realizados como parte de nuestra investigación etnográfica, reconstruiremos las memorias y recorridos biográficos de tres mujeres indígenas que trabajan en ámbitos escolares, en función de lo cual abordaremos tópicos tales como: competencias lingüísticas, trayectorias escolares, vínculo con lo religioso, relaciones de género, transmisión de prácticas culturales, lazos con la familia y comunidad, entre otros.

Este artículo presenta dos grandes desafíos que nos proponemos como equipo de investigación. En primer lugar, se pondrán en vinculación los procesos educativos de dos provincias con diferentes recorridos en torno a la institucionalización de la EIB. Chaco es una provincia pionera y vanguardista en cuanto al reconocimiento de los derechos indígenas en general, y educativos en particular, a pesar de sus contradicciones. Desde la sanción de la Ley $\mathrm{N}^{\circ} 3258$, en 1987, se desarrollaron experiencias escolares interculturales en las que el rol de los docentes indígenas ha sido protagonista. A su vez, desde ese año, en Chaco se encuentra la primera y más reconocida institución para la formación de maestros indígenas: el Centro de Investigación y Formación para la Modalidad Aborigen (CIFMA) que, como se ampliará luego, es donde se formaron las maestras aquí presentadas. En ese sentido, en Chaco se cuenta con una institucionalización de la EIB que le otorga formalidad a la figura docente. Misiones, por su parte, formalizó la EIB recién en 2005, cuando dio inicio a un Programa Intercultural de Frontera (básicamente con las escuelas lindantes con Brasil), y unos años más tarde se expandieron las iniciativas de educación bilingüe para las poblaciones indígenas locales mediante la incorporación de auxiliares bilingües en las instituciones con matrícula mbyá guaraní. La calificación necesaria para desempeñarse como auxiliar bilingüe es la suma de una capacitación ad hoc y los estudios primarios completos. Estos elementos permiten visualizar las distancias comparativas entre las experiencias que abordaremos. Ambos escenarios contrapuestos son representativos del amplio abanico de experiencias escolares para población indígena que presenta la Argentina y, por lo tanto, habilitan una reflexión general sobre alcances y limitaciones de esta política en la actualidad.

El segundo desafío y aporte de este artículo es de orden teórico-metodológico y refiere a la incorporación, dentro del enfoque etnográfico, de los alcances de la utilización de relatos de vida. Justamente, una de las contribuciones más relevantes de la antropología de la educación ha sido la utilización de la etnografía para la indagación de los procesos formativos, aporte metodológico que actualmente es herramienta de diversas disciplinas en la indagación escolar (Neufeld, 2009; Rockwell, 2009). Siguiendo esta línea, nuestra investigación se basó en el análisis de material construido desde un abordaje etnográfico, en el que hemos incluido las aproximaciones biográficas como un tipo
1. La Dra. Enriz realiza estudios etnográficos con la población mbyá desde el año 2003 y la Lic. Cantore, desde 2014. Mientras que con el pueblo toba/qom trabajan las Dras. Hecht y García Palacios desde 2002 y 2006, respectivamente, y la Lic. Aliata, desde 2005. Los nombres de las mujeres cuyas biografías constituyen el corpus central de este texto han sido cambiados, para mantener el anonimato propio del acuerdo ético de confidencialidad en las investigaciones. 
particular de enfoque para relacionar los procesos sociales e históricos. Mediante la realización de entrevistas abiertas antropológicas como técnica para la producción de relatos de vida (Saltalamacchia, Colón y Rodríguez, 1983; Piña, 1989; Padawer, 2012), realizadas con maestras tobas/qom y mbyá, abordamos una narración detallada de su vida, con énfasis en su historia escolar, sin que la entrevistadora interfiriera con preguntas o comentarios que alterasen el hilo conductor del relato. En cada uno de estos relatos, los sujetos desarrollan un guion propio, en el que establecen relaciones entre eventos, personas, fenómenos, que las investigadoras no establecimos a priori. A su vez, las entrevistadas tienen la libertad de moverse en el registro de modo tal que construyen una temporalidad que les es propia, lo que supone una inmensa riqueza de registro. Por medio de esta metodología es posible captar la narración de experiencias respetando la perspectiva y orientaciones propias de las entrevistadas y aprehender los ejes significativos por medio de los cuales estructuran su experiencia (Appel, 2005).

Este relato se produce en el contexto de un trabajo de campo previo que propicia la disposición de los sujetos para narrar su experiencia en un marco de confianza. La interpretación de lo dicho se realiza también mediante las herramientas que las investigadoras hemos construido desde el trabajo de campo etnográfico previo y que nos permiten poner en un contexto mayor y de un modo particular lo dicho. El método comparativo surge aquí como una condición necesaria para establecer generalidades y singularidades de mujeres en experiencias educativas interculturales. Por último, estos relatos habilitan nuevas relaciones y vínculos con hechos históricos más amplios.

Este trabajo se organiza en cuatro secciones. En la primera, se detallan aportes teóricos de los estudios sobre trayectorias de docentes y dentro del campo de las mujeres indígenas. Luego, se recorren aspectos contextuales de las políticas de EIB en las dos provincias estudiadas y se presenta muy someramente a las docentes indígenas de Chaco y Misiones. En el tercer apartado, se desarrollan los ejes analíticos relevantes para la comprensión de la complejidad de las trayectorias de las mujeres tobas/qom y mbyá guaraní, para luego finalizar con las conclusiones generales.

\section{Aportes teóricos sobre las trayectorias de docentes mujeres indígenas}

Las trayectorias docentes en contextos interculturales han sido objeto de interés de las ciencias de la educación en nuestra región. En algunos casos, se focalizó en las identidades, saberes y prácticas pedagógicas de maestros indígenas, con énfasis en la noción de identidades pedagógicas (Guzmán y Garzón, 2008). En otros, se hizo hincapié en las funciones del maestro y la participación de los pueblos en el diseño y planificación de los programas de EIB, y el papel que desempeñan los maestros en la escuela y la comunidad en tanto agentes de "revitalización cultural" (Ipiña, 1997; Serrano Ruiz, 1998; Remedios y Tafoya, 2001; Córdoba, 2004). En el caso de Córdoba (2004), la autora reconstruye su propia trayectoria como docente rural quechua hablante y reflexiona sobre la escuela y la EIB.

Si bien este interés por parte de las ciencias de la educación se replica en la Argentina, cobran especial relevancia las indagaciones desde el campo de la antropología y educación, por ejemplo, respecto de las transformaciones en el trabajo docente, la cotidianeidad escolar y las nuevas prácticas de los educadores (Batallán, 1998; Rockwell, 2013). A su vez, el ámbito de la educación intercultural se vio renovado a partir de la incor-

2. En el contexto regional contamos con antecedentes muy interesantes en el tema, como son los aportes de Czarny (2008), Ramos (2010) y Zúñiga (2017). poración de estudios sobre la formación y capacitación de los docentes indígenas, los debates acerca de la diversidad lingüística y sociocultural, los procesos identitarios y los múltiples saberes en disputa (Díaz, 2001; Achilli, 2008; Valdez y otros, 2015; Villarreal y Martínez, 2015; Medina, 2015; Hirsch y Serrudo, 2015; Hecht y Schmidt, 2016). ${ }^{2}$ 
Más allá de los numerosos avances en este campo, queda un vasto camino para profundizar en las experiencias formativas de las figuras docentes indígenas, más aún en el caso de las mujeres que se desempeñan como tales, si tenemos en cuenta que, en estas comunidades, a diferencia de lo que parecería suceder en el campo de la educación en general, la docencia no ha sido una profesión necesariamente "feminizada" y se han reservado pocos espacios para las mujeres. En este punto, nos parece importante subrayar que las trayectorias, en el caso puntual que nos ocupa, nos permiten describir, desde una perspectiva que vincula lo etnográfico con lo biográfico, las distintas posiciones que los sujetos ocupan en los espacios sociales, y cuyos sentidos sólo pueden definirse relacionalmente teniendo en cuenta tanto sus capacidades de acción como los condicionamientos sociohistóricos (Cragnolino, 2006; García Palacios y Hecht, 2012; García Palacios y otros, 2016). En tanto las relaciones desiguales de género constituyen uno de estos condicionamientos, resulta fundamental comprender las particulares experiencias que vivieron las maestras indígenas como mujeres en su formación y rol, así como reflexionar sobre la diversidad de espacios formativos que configuran sus identificaciones, desentrañando las desigualdades de género que impactaron sobre sus trayectorias.

En relación con los antecedentes teóricos de los estudios de género en comunidades indígenas, se cuenta con importantes reflexiones (Hirsch, 2008; Castelnuovo, 2015; Gómez, 2017) acerca del lugar asignado a las mujeres indígenas en las etnografías más clásicas. En dichas obras, las mujeres tobas/qom y mbyá son representadas como meras reproductoras culturales (Mètraux, 1935; Braunstein, 1974; Idoyaga Molina, 1977; Susnik, 1983; Cadogan, 1997 [1959]; Schaden, 1998 [1954], entre otros) lo que da cuenta de una imagen fosilizada (Hirsch, 2008) que se corresponde con los imaginarios de los pueblos indígenas como estáticos y homogéneos (Gómez, 2017) y en la cual la división sexual del trabajo es entendida como base de la supervivencia de los grupos. Así, la diferencia sexual aparece como el cimiento para comprender las diferencias entre hombres y mujeres, y para separar en tareas "propias de cada uno" dando por sentado el binarismo sexo-genérico. En consecuencia, lo femenino se liga a la crianza de los niños y al cuidado del grupo doméstico; y lo masculino aparece por fuera del núcleo familiar en representación de la comunidad, y adquiere per se un espacio de negociación y de poder del que las mujeres indígenas quedan excluidas (Cantore, 2014).

Es posible que estas narrativas respondan a los intereses de la antropología en sus inicios: si bien se intentaba recabar las diferencias entre hombres y mujeres (Lamas, 1999), los datos fueron recolectados por medio del diálogo con varones adultos como informantes clave, lo que dejaba en evidencia el androcentrismo antropológico y sus formas de trabajo (Moore, 2009). En tanto su interés no estaba puesto en las mujeres, la mayoría de los trabajos etnográficos dieron por sentado que ellas no se vinculaban con personas por fuera de sus grupos más próximos, con lo cual volvían a quedar "fuera de la historia" (Scott, 2010). Al soslayar las relaciones interétnicas de las que ellas son parte, no pudieron dar cuenta de cómo se modifica la construcción social e individual de género (Gómez, 2013). Estas representaciones ahistóricas y al margen de las relaciones de poder contribuyen, finalmente, con la condena de cualquier intento de las mujeres por transformar sus vidas (Hernández y Canessa, 2012).

Si bien es necesario reconocer que gran parte de los lugares de liderazgo y de vinculación hacia fuera de los grupos no han sido ocupados por mujeres, esto está más relacionado con una yuxtaposición de lógicas de género indígenas y occidentales que las excluyen de estos ámbitos, más que con una "actitud conservadora" de las mujeres (Gómez, 2013). Las intervenciones del mercado, el Estado, los vínculos con la sociedad envolvente, las ONG, entre otros, conlleva, según Segato (2011), a una "superinflación de los hombres"; es decir, su poder se incrementa dentro de las comunidades por ocupar el papel de intermediarios con el mundo exterior. 
Aunque gran parte de estas construcciones sexo-genéricas occidentales e indígenas sigan vigentes, esto no significa que las mujeres no discutan con ello, lo (re)signifiquen o innoven sobre los significados culturales recibidos; sino que más bien aportan conocimientos y acciones que "pocas veces se reconocen como contribuciones al desarrollo cultural, político y económico" (Vásquez García, 2012: 320). En este sentido, concordamos con Mohanty (2008) en que la producción académica debe tener en cuenta los contextos locales particulares en los que se expresa la capacidad de agencia de las mujeres, lo cual vuelve a subrayar la importancia de los enfoques que reconocen en sus trayectorias, tanto los condicionamientos sociales, como su agencia.

Muchas mujeres indígenas experimentan transformaciones que les permiten disputar con modelos hegemónicos de género, tal como se verá más adelante en el caso de las que construyen otro lugar dentro de sus comunidades y logran insertarse en espacios escolares y de referencia, habilitados, a su vez, por el contexto histórico más amplio del panorama nacional y local en materia de políticas educativas.

\section{Relatos de las docentes indígenas en la EIB de Chaco y Misiones}

El proceso de consolidación de un sistema educativo que contemple los grupos considerados minorías étnicas en Argentina ha pendulado entre propuestas universales de carácter homogeneizador y políticas focalizadas (Alonso y Díaz, 2004; Novaro, 2006; Hecht, 2007; Hecht y otros, 2016). Se reconoce a las primeras como aquellas que respondieron a modelos escolares monolingües, que negaban y soslayaban las especificidades lingüístico-culturales bajo propuestas homogeneizadoras. Durante el siglo XIX y mediados del XX se impuso a indígenas y migrantes alfabetizarse en español, la lengua hegemónica del Estado, e invisibilizar en la escuela los saberes y contenidos culturales propios.

En cambio, las propuestas focalizadas dieron cuenta de la inserción de experiencias bilingües en programas concebidos como compensatorios y surgieron en las últimas décadas del siglo XX en el marco de los diseños pedagógicos de EIB con el propósito explícito de respetar la diversidad lingüístico-cultural. Lamentablemente, la histórica desigualdad e inferiorización de las poblaciones indígenas no logró ser dimensionada, lo que ha derivado en la reproducción de la subordinación, pese al supuesto objetivo de lograr una igualdad de oportunidades (García Palacios y otros, 2016). En definitiva, mientras que los modelos homogeneizadores responden a los lineamientos sarmientinos, las propuestas focalizadas formaron parte del ideario de las políticas neoliberales que encubren las desigualdades socioeducativas.

El cambio más relevante se produjo con la Ley de Educación Nacional № 26.206 (2006), que ubica a la EIB como una modalidad del sistema educativo para los pueblos indígenas en todos los niveles escolares. No obstante, a estas políticas les queda mucho camino por recorrer. Tanto especialistas como activistas indígenas cuestionan el alcance de muchas de las acciones y demandan la producción de índices que permitan ponderar su impacto en la escolarización y retención escolar. Los aspectos más complejos y controversiales en la práctica son el bilingüismo - por el hecho de que muchas lenguas nativas están siendo paulatinamente reemplazadas por el español-y los alcances y límites de la interculturalidad en la cotidianeidad de las escuelas (García Palacios, Enriz y Hecht, 2014; Padawer y otros, 2017). Algunas de estas cuestiones serán subrayadas en este artículo, siguiendo las trayectorias de las mujeres que presentaremos someramente a la manera de una muy breve introducción.

Sandra es una mujer qom que tiene 39 años, nació y creció en Colonia Aborigen, Chaco, donde vive actualmente y se desempeña como maestra en una escuela primaria. A los 
17 años fue a Sáenz Peña a estudiar en el terciario CIFMA, y formó parte de la segunda promoción egresada de la institución. Fue allí donde, aun sin ser hablante del qom, se relacionó por primera vez con otros jóvenes que sí hablaban la lengua. Sus padres no terminaron la escuela primaria.

Inés es una mujer qom que tiene 27 años, que nació, creció y vive actualmente en uno de los barrios tobas de Sáenz Peña, Chaco. Allí realizó desde sus estudios primarios hasta su carrera docente en el CIFMA. Inés habla qom desde pequeña, es la primera maestra bilingüe intercultural de jornada completa de la provincia y se desempeña en una escuela primaria. Sus padres eran cosecheros y no asistieron a la escuela. Ella congrega en la Iglesia Pentecostés Qompi, radicada en el barrio donde vive y presidida por su padre, que es pastor.

Karina es una mujer mbyá que tiene 27 años y se desempeña como auxiliar bilingüe en una escuela primaria en su comunidad en Misiones, después de que ella se propusiera y la comunidad la admitiera como tal. Es hija de una familia prestigiosa, ya que su padre fue el cacique durante mucho tiempo y su abuela fue una referente religiosa muy importante. Allí asistió a una escuela primaria de jornada completa de la Iglesia católica, pero luego se trasladó a un secundario en el pueblo más cercano, trayecto que no pudo terminar. Ninguno de sus padres está escolarizado.

A lo largo del escrito, ampliaremos estas breves descripciones con otros datos significativos, a medida que avancemos en el análisis de aquellos rasgos más destacados (ya sea por su contraste o por la continuidad que presenten los distintos relatos).

\section{Trayectorias educativas de maestras tobas/qom y mbyá}

En este apartado, se desarrollarán los ejes analíticos que han resultado relevantes para la comprensión de la complejidad de las trayectorias de las mujeres con las que trabajamos. En primer término, nos concentraremos en reflexiones sobre los condicionamientos sexo-genéricos en un contexto intergeneracional, en especial sobre el hecho de ser mujer, ser indígena y querer estudiar. Luego, el foco se concentrará en la transmisión cultural de saberes significativos como los vinculados al ámbito religioso y lingüístico. Por último, se revisan los procesos de identificación en la formación y el rol docente.

\section{Ser mujer, ser indígena y querer estudiar: cambios intergeneracionales}

Un aspecto que vuelve relevantes a las historias de las mujeres que recuperamos en este análisis es que, en todos los casos, sus trayectorias de formación son más extensas que las del promedio de mujeres de sus comunidades. El mayor nivel de escolarización alcanzado parece habilitarlas para manejarse con más solvencia en ciertas actividades propias de su lugar de referentes, ya que, en todos los casos, desempeñar un rol en el marco de la escuela implica una referencia y valoración singular del grupo.

Los estímulos y acompañamientos de la familia en las trayectorias se repiten en los casos de análisis; en algunos, con referencias concretas al propio grupo étnico, y en otros, a personas de confianza. En general, se menciona a un/a familiar, maestro/a o religioso/a que presta apoyo económico y afectivo y que las estimula en los estudios. Así, por ejemplo, en el caso de Inés, el apoyo central fueron sus padres, cosecheros, que migraban por trabajo, lo cual fragmentaba las trayectorias escolares de sus hijos. Al ser ella la hija menor, fue la menos afectada por esta situación, pues sus padres anhelaban que fuera docente y así fue. Sandra, por su parte, se decidió a estudiar en el CIFMA a los 17 años, incentivada por un cura párroco miembro de ENDEPA (Equipo Nacional de Pastoral Aborigen). 
La posibilidad de tejer redes que van más allá del espacio escolar pero que impactan en los itinerarios escolares les permitió a estas mujeres sostener gran parte de los aprendizajes que llegan a ser requeridos y valorados por los mecanismos escolares (Rockwell, 2011). De este modo, no se trata solamente de redes de relaciones que facilitan la asistencia, sino que también proporcionan ayuda en la alimentación, vestimenta y en la organización de las tareas que incluyen horarios de estudio.

En los relatos, además, se alude a mujeres significativas (madres, abuelas) que han sido referentes del colectivo genérico por la fortaleza que impregnaron en sus prácticas. Esas mujeres, que no lograron formación de tipo escolar, pudieron desempeñarse en roles importantes dentro del propio grupo, como líderes espirituales y de referencia. Las ventajas están representadas por la posibilidad de acceder a la escuela, algo a lo que no pudieron acceder anteriores generaciones. Por tanto, en el relato de Karina aparece la necesidad de generar, en las comunidades, escuelas con "mayor exigencia", que formen a los y las estudiantes para emprender y superar desafíos escolares mayores.

Otra cuestión para subrayar en las trayectorias escolares es el entrecruzamiento de las asignaciones desiguales de género con las marcaciones étnicas y de clase. Incluso las trayectorias educativas de larga duración - como las de Inés o Sandra- parecieran destacarse sobre un trasfondo de desigualdades que, más allá de afectar en términos generales a los colectivos marcados étnicamente, también diferencian genéricamente a varones de mujeres, lo cual enfatiza la particularidad de ellas por haber finalizado sus estudios, siendo indígena y mujer. En las entrevistas aparece la tensión entre estudiar y formar una familia: Karina, por ejemplo, manifiesta que las mujeres mbyá no estudian porque "se acompañan desde chicas o tienen hijos muy temprano" (Karina, junio 2008).

La recurrente exclusión de las mujeres de las relaciones interétnicas más valoradas por el grupo - ya referida - contribuye con la consolidación de un modelo hegemónico de género que les asigna a ellas la preservación de ciertos elementos culturales. Estudiar implica, en todos los casos analizados, salir de la comunidad, del propio núcleo de referencia, del ámbito doméstico, y desempeñarse en una exterioridad con actores nuevos. En las trayectorias analizadas, para concretar los estudios es condición vincularse con instituciones que se ubican lejos del hogar. Para Karina, la experiencia actual de los jóvenes que viven la comunidad y asisten a la "escuela del pueblo" se frustra cuando "esperan que los traten distinto que a los otros, porque están acostumbrados a que acá —en la escuela de la comunidad - los cuidemos demasiado" (Karina, junio 2008).

En términos materiales, estas mujeres forman parte de un mismo sector social, privado de recursos para su desarrollo académico, lo que coloca a sus trayectorias en lugares de mayor protagonismo. Tanto los asentamientos tobas como las comunidades mbyá a que aludimos en este texto son ámbitos de ventajas relativas para la escolaridad, pero que a la vez enfrentan enormes dificultades de recursos. Las ventajas están representadas por la posibilidad de acceder a la escuela, de la que no disponían anteriores generaciones. Las limitaciones quedan manifiestas cuando expresan la alternativa de trabajar en el yerbal u otra cosecha a temprana edad. A su vez, tanto Inés como Karina quisieron estudiar otras cosas, pero por falta de recursos, no pudieron, y las tareas docentes se volvieron una buena opción.

La atención particular sobre la población indígena se manifiesta claramente en las reflexiones de Inés, quien protestó junto con "un grupo de hermanas indígenas" (Inés, octubre 2016) por las condiciones edilicias y, como parte de una estrategia del Estado para mejorar el nivel de participación de los chicos en dicha escuela, recorrió las casas para anotar a los/as alumnos/as. Estas acciones denotan el valor que ella le da a su incorporación en la escuela del barrio. Por tanto, la escuela que estas mujeres quieren producir forma parte de un relato en el que se contempla la particularidad lingüística, 
los/as indígenas se encuentran incluidos en las aulas también como docentes y la comunidad tiene la posibilidad de condicionar esa incorporación a partir de cierto tipo de selección de los miembros habilitados a cumplir con un rol docente.

\section{Las mujeres y la transmisión cultural}

En la construcción de las identificaciones étnicas, la lengua suele indicarse como uno de los diacríticos más importantes. La lengua mbyá-guaraní, ${ }^{3}$ en amplio contraste con el toba/qom, como veremos más adelante, presenta altas tasas de monolingüismo en la población infantil, asentada mayoritariamente en contextos rurales. Tal es el caso de Karina, que, en la narración de su historia, cuenta que era monolingüe en la lengua nativa antes de ingresar a la escuela primaria de su comunidad. No obstante, a pesar de la vitalidad de la lengua indígena en Misiones, sorprende que la EIB aún sea una política incipiente en cuanto a su intervención con las poblaciones indígenas (Enriz, 2011). A este respecto, en el relato sobre su escolarización, Karina da cuenta de dos aspectos nodales. Por un lado, su generación fue la primera de su familia en ir a la escuela. Por otro, indica que esta experiencia transcurrió en una de las primeras escuelas de la provincia instaladas en el predio de una comunidad mbyá. Esta institución educativa pertenecía a la Iglesia católica ${ }^{4}$ y no contaba con personal indígena. Antes de que existiera esa escuela, que cuenta con 28 años de antigüedad, algunos niños de la comunidad se trasladaban a la escuela del pueblo; situación que a ella le tocó vivir cuando asistió a la secundaria: recuerda con pesar que fue al secundario del pueblo más cercano, y que para llegar debía caminar unos cinco kilómetros. Como le resultó muy dificultoso, luego se inscribió en una EFA (Escuela de la Familia Agrícola, de permanencia), a la que sólo asistían siete indígenas, entre quienes ella era la única mujer.

Con respecto a la lengua toba/qom, ${ }^{5}$ muchas investigaciones dan cuenta de la gran variabilidad en cuanto a su vitalidad y uso cotidiano, por lo que es muy difícil establecer generalizaciones homogéneas sobre su situación sociolingüística. A grandes rasgos, los estudios coinciden en la apreciación de que está atravesando procesos de desplazamiento por el español en la mayoría de los eventos comunicativos cotidianos en los que participan los niños y las niñas de contextos urbanos (Messineo, 2003; Hecht, 2010). En ese sentido, las experiencias lingüísticas de las dos mujeres tobas/qom son un reflejo de la enorme variabilidad intralingüística, ya que mientras Inés es hablante fluida, Sandra recién aprendió la lengua indígena cuando estudiaba en el terciario.

Cabe señalar que estos escenarios sociolingüísticos se evidencian a pesar de los avances en las legislaciones sobre EIB con el fin de mantener las competencias lingüísticas en toba/qom (Hecht, 2015).

Si bien las continuidades y discontinuidades en la transmisión de saberes a las jóvenes generaciones se expresan en diversas dimensiones de la vida social, las referentes mencionan con especial énfasis una preocupación por la "pérdida de la lengua”. Para las mujeres qom, la preocupación se vincula con la variabilidad de sus propias trayectorias y los desafíos actuales que tienen como docentes al enseñar la lengua indígena a niños y niñas que no son hablantes. Contrariamente, en el caso de Karina, se muestra la dificultad con la que se encuentran los y las docentes al tratar de enseñar la lengua mbyá guaraní, que — si bien es muy vital en su uso oral—, no cuenta con una sistematización ni una gramática específica, $y$, por lo tanto, una didáctica adecuada que se actualice en capacitaciones sistemática. Incluso el hecho de recurrir al guaraní paraguayo como estándar para su enseñanza, como sucede en muchos casos, atenta contra las especificidades de esta lengua. Más allá de estos matices entre los contextos, todas las mujeres señalan la importancia del reconocimiento identitario, independientemente de las competencias en la lengua nativa.
3. La lengua mbyá pertenece a la familia lingüística tupi-guaraní y abarca a Argentina, Paraguay y Brasil. Según el Censo Nacional de Población 2010, en Misiones hay 13.000 personas mbyá-guaraní.

4. En un artículo anterior, desarrollamos el rol que han tenido las iglesias en la consolidación de propuestas educativas para poblaciones indígenas en Chaco y Misiones. Hemos analizado el lugar pionero de estas y las limitaciones que conllevaban debido a los objetivos evangelizadores (ver Enriz, García Palacios y Hecht, 2017).

5. La lengua toba pertenece a la familia guaycurú y es una de las lenguas indígenas de Argentina con mayor cantidad de hablantes. Según el Censo Nacional de Población 2010, las personas autorreconocidas como tobas/ qom son 126.967 y residen en Buenos Aires, Chaco y Santa Fe. 
6. El Evangelio es un movimiento sociorreligioso que fusiona aspectos de las propuestas religiosas indígenas con el pentecostalismo.
Ahora bien, en la reconstrucción de las trayectorias educativas es interesante situar el análisis de lo escolar dentro de una trama compleja de instituciones y espacios por donde circulan las personas, y que habilitan sus diversas experiencias formativas. En los relatos de estas mujeres, las iglesias y lo religioso se entrecruzan con sus referencias a la escolarización y con los aprendizajes construidos a lo largo de su vida. Cuando se coteja lo que se conoce y enseña, la escuela, la religiosidad y los "contenidos culturales" de la comunidad aparecen como instancias complementarias: Inés lee la Biblia y esa es una guía para ella, porque esa fe la lleva a sostener la EIB y la formación religiosa; Karina valora muy positivamente que se brinden en la escuela conocimientos en mbyá, canciones y relatos, pero piensa que sería importante también tener un opy (lugar donde se realiza la ceremonia religiosa mbyá), para que esas experiencias continúen allí junto a las palabras de los abuelos.

Incluso mujeres como Inés y Karina cuentan en su linaje con antepasados que son referentes indígenas religiosos y eso no sólo impacta en el prestigio que portan sino también en el acceso a un campo más amplio de saberes, ya que, para ellas, la religión ofrecería la posibilidad para la continuidad de ciertos "contenidos culturales"; sobre todo para el caso toba/qom, en el que el Evangelio ${ }^{6}$ incorpora en una nueva creación elementos nativos "tradicionales" que de otro modo podrían perder continuidad y en el que los distintos aprendizajes que suelen valorarse entre las personas que se "han entregado al Evangelio" guardan un estrecho vínculo con saberes ponderados para el liderazgo, como la habilidad con la palabra (Ceriani y Citro, 2005; García Palacios, 2012). En el caso de Inés, por ejemplo, un signo de su rol de referencia fue vencer la timidez y lograr hablar "cuando hay que hablar" (Inés, octubre 2016).

No aparece tan manifiesta la cuestión de la laicidad de las propuestas escolares, sino más bien el valor que el fenómeno religioso presenta en las experiencias extraescolares. Respecto de Karina, por ejemplo, la escuela de su comunidad es de gestión privada y de administración católica. Esto no interviene en su propia religiosidad, que se manifiesta explícitamente mbyá.

Como hemos mencionado, adjudicar a las mujeres el rol de protección de la lengua, así como el cuidado de la transmisión de saberes, supone reforzar un rol de género monolítico y ahistórico, pero resulta, además, en los casos analizados, un valor que se porta. Estas mujeres recuperan ese mensaje en términos de oportunidad para hacer de ellas, de su vida y sus comunidades algo distinto. Estudiar, como algo opuesto a lo esperado por el modelo enunciado, es para ellas una herramienta de fortalecimiento propio y de sus comunidades; una oportunidad.

De hecho, no se trata sólo del desempeño laboral: mientras estudia, Sandra es voluntaria en actividades de fortalecimiento de su comunidad, alfabetizando adultos y colaborando con niños con dificultades en su escolaridad.

En todos los casos, las búsquedas profesionales implicaban un compromiso social: ser enfermera, ser abogada, ser docente fueron parte de sus intereses. En todos los casos, el rol implicaba una "traducción" de conocimientos institucionales de relevancia para las comunidades.

\section{Procesos de identificación en la formación y el rol docente}

Los cambios en las políticas educativas y el proceso de la institucionalización de la EIB han impactado de manera diversa según se trate de una u otra generación y de uno u otro contexto dentro de las poblaciones con las que trabajamos, tal como fue haciéndose evidente en los anteriores acápites. 
Así, por ejemplo, Sandra representa claramente la vivencia de muchas mujeres que no han recibido en su infancia y adolescencia lo que ella llama "la herencia cultural de la comunidad" (Sandra, octubre 2015). Ella pertenece a una primera o segunda generación cuyas experiencias formativas - aun al vivir con la familia y asistir a una escuela en la misma comunidad- muestran cierta ausencia en la transmisión de la lengua indígena y/o en lo relativo a ciertos aspectos socioculturales vinculados por ella con lo indígena. Esto se evidencia particularmente en Sandra ya que en la comunidad donde vive es donde se produjo la matanza de Napalpí. Es decir, dentro de su familia y en la escuela no se mencionaba la pertenencia a una comunidad indígena; tampoco se hablaba la lengua ni de la historia. Como ella sostiene, "si mencionabas que eras perteneciente a una comunidad indígena tenías menos posibilidad de conseguir trabajo o realizar estudios terciarios" (Sandra, octubre 2015). En este punto señalábamos anteriormente que sus abuelos y padres no habían tenido las mismas oportunidades de escolarización que ella. No obstante, en una experiencia singular, estas situaciones se revierten al transitar los estudios terciarios: "En el terciario me encontré con todo, con la identidad, con la lengua" (Sandra, octubre 2015). La visibilidad y transformación de la identificación étnica, la historia y la lengua indígena que transita Sandra - al igual que muchas de estas mujeres durante sus estudios para ser maestras indígenasmarca un hito en las trayectorias personales. Esto resulta del todo congruente con lo planteado por investigaciones que abordan cómo los estudios superiores impactan en las identificaciones de estudiantes de comunidades indígenas (Czarny, 2008; Paladino, 2010; Ossola, 2015), en algunos casos contribuyendo también con la problematización de las relaciones sexo-genéricas conocidas (Rea Ángeles, 2011).

De este modo, Sandra menciona que en el terciario "teníamos que investigar acerca del origen de nuestra familia (...) Ahí descubro, me descubro yo y descubro la historia de mi mamá y de mis abuelos" (Sandra, octubre 2015). Si bien en las experiencias personales de Inés y Karina la pertenencia a una comunidad indígena se vivencia desde la propia infancia, la incorporación como docentes también manifiesta una nueva arista en estas identificaciones. Así, por ejemplo, Inés menciona su paso por el terciario como un punto de importancia en su formación: recuerda que mientras estudiaba su padre le construyó un nvique (violín qom) y le enseñó a tocarlo para un trabajo del CIFMA.

El análisis de las identificaciones externas, entendidas como aquellos sistemas de categorización hegemónicos (Brubaker y Cooper, 2001), permite pensar cómo las tipificaciones producidas por los establecimientos estatales condicionan el desempeño de los estudiantes. En muchos casos, se destacan construcciones negativas del indígena efectuadas desde los establecimientos escolares que actuaron como un factor expulsivo y discriminador. Así entre la población toba/qom es frecuente registrar a la escuela de tiempos pasados como "autoritaria, una escuela que segregaba mucho, más a los indígenas, diferente a la de hoy" (Sandra, octubre 2015). El caso de Misiones, expresado en la entrevista de Karina, pone de manifiesto una trayectoria diferente, en la que se sostiene la identidad indígena, aun desde recorridos escolares que sólo se proponen acercar a los niños/as al español y al mercado de trabajo. Es decir, hay un salto entre experiencias que se transitan de modo diferencial en las entrevistas en el pasado y en la actualidad.

Los diferentes trayectos y tipos de formación que presentan las tres mujeres docentes también se deben a los cambios que ha atravesado la formación docente en el devenir del tiempo en el Chaco y Misiones, y muestran como la EIB se fue institucionalizando y expandiendo a través de las décadas. Así, en lo concerniente a su rol como docentes, las tres (al igual que otros/as maestros/as con los que hemos trabajado) sienten que su formación y la de sus colegas actuales ha sido despareja, en tanto algunas han recibido una formación como auxiliares docentes y otras como profesores bilingües e interculturales. 
Otro aspecto relacionado con su práctica laboral, y que se desprende de lo analizado anteriormente, remite a los cambios en los perfiles sociolingüísticos de los/as estudiantes indígenas y cómo eso impacta en las demandas que se les efectúan profesionalmente. Desde la implementación de la EIB, lejos de aumentar los hablantes de lenguas indígenas, se evidencia un acelerado proceso de desplazamiento lingüístico a favor del español, sobre todo en las ciudades donde reside un gran porcentaje de población toba (Hecht, 2010). Por lo tanto, uno de los nuevos desafíos que señalan las maestras tobas/ qom en relación con su función actual como docentes es enseñar las lenguas indígenas como segundas lenguas a niños/as que se autorreconocen como indígenas. Es decir, su rol pasó de alfabetizar en lengua indígena a revitalizar, recuperar la lengua. En ese sentido, sienten que hay cierta falta de contenido curricular a enseñar: su función, según ellas mismas, es concientizar a los niños y niñas de que son indígenas, y la lengua es un diacrítico importante de esa identificación. En otros contextos donde la lengua está aún vital, como puede ser el caso de Misiones, el desafío profesional radica en buscar estrategias pedagógicas para enseñar incluyendo la lengua indígena en los distintos niveles escolares.

\section{Conclusiones}

En este artículo se mostraron la textura, la complejidad y las contradicciones de las trayectorias educativas de las maestras tobas/qom de la provincia del Chaco y mbyáguaraní de Misiones. Estas mujeres han logrado posicionarse como sujetos relevantes y decisivos en la cotidianeidad educativa actual de sus comunidades. Incluso logran revertir la imagen clásica y estereotipada de las mujeres indígenas como ceñidas al hogar y por fuera de los espacios públicos.

La compleja desigualdad material y simbólica que enfrentan las comunidades indígenas ante el sistema educativo no fue experimentada por estas mujeres únicamente como un límite, sino que atravesaron procesos de transformación de sí mismas y a través de ellas, de sus comunidades. Yo quiero estudiar por mi comunidad es la síntesis más clara de esta apuesta: formarse para transformarse y transformarse para brindarle nuevas oportunidades al grupo del que forman parte. Nuevas oportunidades en un campo específico como el educativo, que permite replicar al infinito las posibilidades de transformación en cada uno de esos/as niños/as con los/as que se comprometen en la tarea cotidiana. Así, la escolaridad es un campo al que siguen apostando las mujeres indígenas. Como representantes de grupos etnolingüísticos alterizados, la mayor apuesta se aloja en la construcción de relaciones sociales más justas, en las que el respeto y la valoración de las diferencias sean más un hecho que un discurso.

En este artículo optamos por un abordaje comparativo, ya que nos parece pertinente vincular situaciones sociolingüísticas y escolares diversas, con el fin de ir delineando poco a poco el papel que desempeñan figuras clave como las maestras indígenas dentro de los procesos de escolarización. Por medio de estas historias, nos propusimos poner en vinculación situaciones educativas interculturales que se desarrollan con distinta sistematicidad y permanencia en el tiempo.

En ese sentido, el enfoque comparativo nos permitió abordar aspectos comunes y singularidades. En todas estas mujeres, hemos encontrado un fuerte interés por la lengua indígena y una valoración positiva de su incorporación en la dinámica escolar. Incluso al tratarse de mujeres con trayectorias familiares muy diferentes respecto de la lengua y con accesos muy desiguales al español. En este marco, abordamos los diferentes 
impactos de instituciones sociales como la familia, la escuela, la iglesia, etc., sobre las representaciones, usos e ideologías lingüístico-culturales.

En línea con el reconocimiento de la antropología feminista acerca de la multiplicidad de las trayectorias y experiencias de vida de cada una de las mujeres, lo que resulta en la gran heterogeneidad en las expresiones de "ser mujer" (Moore, 2009), este trabajo permite visibilizar experiencias del ser mujer indígena que se distancian del modelo sexo-genérico hegemónico. Nos interesó especialmente puntualizar el papel fundamental que les cabe a estas mujeres en tanto docentes en la implementación de la EIB: salir a buscar a los niños/as a sus casas, contenerlos en la experiencia, comprenderlos en el propio cuerpo. Como indica Nucinkis (2007, en Serrudo, 2010), sin el apoyo y convencimiento de maestros y maestras, la EIB corre el riesgo de quedarse en el discurso institucional en lugar de ser la propuesta de transformación radical que se desea y necesita fomentar. Las historias de vida de estas mujeres permiten también advertir la real dimensión que el sistema educativo tiene como puntapié en la vinculación de las poblaciones indígenas con el Estado. A la presencia de escuelas deviene luego la asistencia sanitaria; en ocasiones, el acceso a planes sociales, asistencia alimentaria, entre otras intervenciones.

Por último, este texto se sostuvo sobre relatos que no sólo han manifestado ideas contradictorias, agudas, complejas, sino un inmenso caudal de sentimientos que se expresaron en las voces de estas mujeres que se atreven a pensar para ellas un destino singular; y para su comunidad, un futuro promisorio. 


\section{Q Referencias bibliográficas}

》 ACHILLI, Elena. 2008. “Formación docente e interculturalidad”. Diálogos Pedagógicos, 12: 121138.

》 ALIATA, Soledad. 2017. "Reflexiones sobre la figura del docente en contextos interculturales en Chaco". Revista Educación, Lenguaje y Sociedad, 14(14). https://cerac.unlpam. edu.ar/index.php/els/article/view/2212/2209. (30 de septiembre de 2017).

» ALONSO, Graciela y DíAZ, Raúl. 2004. “¿Es la educación intercultural una modificación del statu quo?”. En: R. Díaz y G. Alonso (Comps.). Construcción de espacios interculturales. Buenos Aires: Miño y Dávila. pp. 75-96.

» APPEL, Michael. 2005. "La entrevista autobiográfica narrativa: Fundamentos teóricos y la praxis del análisis mostrada a partir del estudio de caso sobre el cambio cultural de los Otomíes en México". Forum Qualitative Sozialforschung / Forum: Qualitative Social Research 6(2): 1-27.

》 BATALLÁN, Graciela. 1998. “¿Puede la docencia ser una profesión? Contradicciones en el marco de la escuela democrática". Revista de la Academia, 3: 123-134.

" BRAUNSTEIN, José. 1974. "Matrimonio y familia entre los matacos". Cuadernos Franciscanos, 35: 72-79.

» BRUBAKER, Rogers y COOPER, Frederick. 2001. “Más allá de 'identidad””. Apuntes de Investigación del CECYP, 7: 30-67.

» CADOGAN, León. 1997 [1959]. Ayvu-Rapyta. Textos míticos de los Mbyá-guaraní del Guairá. Asunción: Fundación León Cadogan, Centro de Estudios Antropológicos de la Universidad Católica/Centro de Estudios Paraguayos Antonio Guasch.

» CANTORE, Alfonsina. 2014. "Antropología y la desnaturalización del sistema de salud: sobre las concepciones de enfermedad de comunidades mbyá de Misiones”. En Letra, 1, número extraordinario, 142-163.

»CASTELNUOVO, Natalia. 2015. Mujeres guaraníes y procesos de participación política en el noroeste argentino. Argentina: Antropofagia.

»CERIANI CERNADAS, César y Silvia CITRO. 2005. "El movimiento del evangelio entre los Toba del Chaco argentino. Una revisión histórica y etnográfica”. En: B. Guerrero Jiménez (Comp.). De Indio a Hermano. Pentecostalismo indígena en América Latina. Iquique: Ediciones Campus. pp. 111-170.

" CÓRDOBA, Galvina. 2004. No le enseñan las cosas para pasar la vida, solo le enseñan a leer y escribir. Reflexiones sobre la escuela. http://www.ibs-treatments.org/project/etext/ Ililas/claspo/rtc/o059.pdf. (29 de septiembre de 2017).

»CRAGNOLINO, Elisa. 2006. "Trayectorias sociales y apropiación de la cultura escrita en un grupo de mujeres de Córdoba”. VIII Congreso Argentino de Antropología Social. Salta: Universidad Nacional de Salta.

»CZARNY, Gabriela. 2008. Pasar por la escuela: indígenas y procesos de escolaridad en la ciudad de México. México: Universidad Pedagógica Nacional.

" DÍAZ, Raúl. 2001. Trabajo docente y diferencia cultural. Lecturas antropológicas para una identidad desafiada. Buenos Aires: Miño y Dávila. 
» ENRIZ, Noelia. 2011. “Fronteras de la EIB (Educación Intercultural Bilingüe)”. En: E. Loncon y A. C. Hecht (Comps.). Educación Intercultural Bilingüe en América Latina y el Caribe: balances, desafíos y perspectivas. Santiago de Chile: Fundación Equitas. pp. 352-361.

» ENRIZ, Noelia y GARCÍA PALACIOS, Mariana. 2008. “Deviniendo Kuña va'era”. En: S. Hirsch (Coord.). Mujeres indígenas de la Argentina. Buenos Aires: Biblos. pp. 205-230.

» ENRIZ, Noelia, GARCÍA PALACIOS, Mariana y HECHT, Ana Carolina. 2017. “Llevar la palabra. Un análisis de la relación entre las iglesias y la escolarización de niños indígenas tobas/qom y mbyá-guaraní de Argentina”. Universitas Humanística, 83: 187-212.

» GARCÍA PALACIOS, Mariana. 2012. Religión y etnicidad en las experiencias formativas de los niños y niñas de un barrio toba de Buenos Aires. Tesis de Doctorado, Facultad de Filosofía y Letras, Universidad de Buenos Aires.

» GARCÍA PALACIOS, Mariana y HECHT, Ana Carolina. 2012. "El entramado de espacios educativos. Posibles aportes de la incorporación de relatos de vida en la etnografía". $2^{\circ}$ Seminario/Taller de Antropología y Educación. Rosario: Universidad Nacional de Rosario.

» GARCÍA PALACIOS, Mariana, ENRIZ, Noelia, HECHT, Ana Carolina. 2014. "Niños y niñas en las fuentes etnográficas sobre poblaciones indígenas (qom-mbyá)”. Revista Papeles de Trabajo, 28: 1-18.

» GARCÍA PALACIOS, Mariana y HECHT, Ana Carolina. 2017. “Experiencias formativas de mujeres referentes tobas/qom". X Seminario Internacional Políticas de la Memoria, Buenos Aires: Centro Cultural de la Memoria Haroldo Conti.

» GARCÍA PALACIOS, Mariana, PADAWER, Ana, HECHT, Ana Carolina y NOVARO, Gabriela. 2016. "Mujeres indígenas: trayectorias educativas de tres referentes comunitarias en Argentina”. En: G. Novaro, A. Padawer y A. C. Hecht (Coords.). Educación, pueblos indígenas y migrantes. Avances desde México, Brasil, Bolivia, Argentina y España. Buenos Aires: Biblos. pp. 161-198.

» GÓMEZ, Mariana. 2013. “Modernidad y género en mujeres indígenas tobas (qom) del Chaco centro-occidental, Argentina: algunas reflexiones”. En: J. Gregor-Strobele y D. Wollrad (Coords.). Espacios de género: Adlaf Congreso Anual 2012. Buenos Aires: Nueva Sociedad. Fundación Friedrich Ebert Adlaf. pp. 288-301.

» GÓMEZ, Mariana. 2017. "La mirada cosmologicista sobre el género de las mujeres indígenas en la antropología del Chaco argentino: una crítica”. Corpus, 7(1). https://corpusarchivos.revues.org/1765. (15 de septiembre de 2017).

» GUZMÁN, Elizabeth y GARZÓN, Lilia. 2008. “Historias y trayectorias de maestras y maestros indígenas en el Cauca: ¿la etnoeducación, posibilidad para Otras educaciones?". Revista Educación y Pedagogía, 50: 81-97.

» HECHT, Ana Carolina. 2007. "Pueblos indígenas y escuela. Políticas homogeneizadoras y políticas focalizadas en la educación argentina”. Políticas Educativas, 1(1): 183-194.

» HECHT, Ana Carolina. 2010. Todavía no se hallaron hablar en idioma. Procesos de socialización lingüística de los niños en el barrio toba de Derqui (Argentina). Múnich: Lincom Europa.

» HECHT, Ana Carolina. 2015. "Trayectorias escolares de maestros Toba/qom del chaco". Cuadernos del Instituto Nacional de Antropología y Pensamiento Latinoamericano, 24: 1-12.

»HECHT, Ana Carolina, ENRIZ, Noelia y GARCÍA PALACIOS, Mariana. 2016. "Reflections on the Concept of Interculturality in the Current Educational Debate in Argentina". Intercultural Education, 27(3): 1-15.

» HECHT, Ana Carolina y SCHMIDT, Mariana. 2016. “La capacitación de auxiliares bilingües en el Chaco salteño”. En: A. C. Hecht y M. Schmidt (Comps.). Maestros de la Educación Intercultural Bilingüe. Buenos Aires: Novedades Educativas. pp. 223-238. 
» HECHT, Ana Carolina, GARCÍA PALACIOS, Mariana, ENRIZ, Noelia y DIEZ, María Laura. 2016. "Interculturalidad y educación en Argentina: reflexiones a propósito de un concepto polisémico”. En: G. Novaro, A. Padawer y A. C. Hecht (Comps.). Educación, pueblos indígenas y migrantes en Argentina, Brasil, México y España. Buenos Aires: Biblos. pp. 43-63.

" HERNÁNDEZ, Aída y CANESSA, Andrew. 2012. “Identidades indígenas y relaciones de género en Mesoamérica y la Región Andina”. En: A. Hernández y A. Canessa (Eds.). Complementariedades y exclusiones en Mesoamérica y los Andes. Ecuador: Editorial Universitaria Abya-Yala. pp. 10-42.

» HIRSCH, Silvia. 2008. "Maternidad, trabajo y poder: cambios generacionales en las mujeres guaraníes del noroeste argentino”. En: S. Hirsch (Coord.). Mujeres indígenas en Argentina. Cuerpo, trabajo y poder. Buenos Aires: Biblos. pp. 231-252.

» HIRSCH, Silvia y SERRUDO, Adriana. 2015. "Trayectorias de la docencia en contextos interétnicos: la labor de los docentes wichí y guaraní en Salta”. En: A. C Hecht y M. A. Schmidt (Comps.). Maestros de la Educación Intercultural Bilingüe. Buenos Aires: Novedades Educativas. pp. 179-198.

»IDOYAGA MOLINA, Antilde. 1977. “Aproximaciones hermenéuticas a las nociones de concepción, gravidez y alumbramiento entre los pilagá del Chaco central”. Scripta Ethnológica, 4(2): 76-98.

» IPIÑA, Enrique. 1997. “Condiciones y perfil del docente de educación intercultural bilingüe”. Revista Iberoamericana de Educación, 13: 99-109.

» LAMAS, Marta. 1999. Usos, dificultades y posibilidades de la categoría género. Papeles de Población, 5(21): 147-178.

» LÓPEZ, Luis Enrique y KÜPER, Wolfgang. 200o. La educación intercultural bilingüe en América Latina: balance y perspectivas. Cochabamba: Programa de Formación en Educación Intercultural Bilingüe para los Países Andinos (PROEIB Andes).

» MEDINA, Mónica. 2015. "Representaciones sobre las funciones de los docentes indígenas en la modalidad EIB de Resistencia”. En: A. C Hecht y M. A. Schmidt (Comps.). Maestros de la Educación Intercultural Bilingüe. Buenos Aires: Novedades Educativas. pp. 161-178.

» MESSINEO, Cristina. 2003. Lengua toba (guaycurú): aspectos gramaticales y discursivos. Múnich: Lincom Europa.

» MÈTRAUX, Alfred. 1935. "La mujer en la vida social y religiosa de los indios chiriguanos”. En: Actas del $26^{\circ}$ Congreso Internacional de Americanistas, Tomo 1. Sevilla: ICA. pp. 416-430.

» MOHANTY, Chandra. 2008. “Bajo los ojos de occidente. Academia feminista y discursos coloniales”. En: Hernández Castillo, R. A. y L. Suárez Navaz (Coord.). Descolonizando el feminismo. Teorías y prácticas desde los márgenes. Madrid: Cátedra. pp. 117-164.

" MOORE, Enriqueta. 2009. Antropología y feminismo. Madrid: Cátedra.

» NEUFELD, María Rosa. 2009. “Antropología y Educación en el contexto argentino”. VIII Reunión de Antropología del Mercosur. Buenos Aires: UNSAM.

» NEUFELD, María Rosa y THISTED, Jean Ariel (Comp.). 1999. De eso no se habla. Los usos de la diversidad sociocultural en la escuela. Buenos Aires: Eudeba.

» NOVARO, Gabriela. 2006. “Educación intercultural en la Argentina: potencialidades y riesgos”. Cuadernos Interculturales, 4(7): 49-6o.

" NOVARO, Gabriela y PADAWER, Ana. 2013. "Identificaciones étnico-nacionales y procesos de legitimación del saber en grupos indígenas y migrantes en Argentina”. Revista Século XXI, 3(1): 1-38. 
» OSSOLA, Macarena. 2015. “Entre permisos y ejemplos. Reconfiguraciones familiares entre los jóvenes universitarios wichí en el noroeste argentino”. Cuicuilco, 22(62): 75-90.

»PADAWER, Ana. 2012. “'Por los hijos hay que seguir dándole para adelante, enseñarles’: una narración biográfica sobre experiencias formativas en contextos rurales y sentidos de la escuela”. En: M. Menna Barreto (Ed.). Diversidade: Culturas, Ruralidades, Migração, Formação e Integração social. Porto Alegre: Pontifícia Universidade Católica de Rio Grande do Sul. pp. 27-54.

» PADAWER, Ana, ARTIEDA, Teresa, BORTON, Laureano y ALMIRÓN, Soledad. 2017. “Alfabetización y evangelización. Intervenciones educativas hacia los qom en el marco de congregaciones religiosas”. En: G. Novaro, L. Santillán, A. Padawer y L. Cerletti (Coords.). Niñez, regulaciones estatales y procesos de identificación. Experiencias formativas en contextos de diversidad y desigualdad. Buenos Aires: Biblos. pp. 187-215.

» PALADINO, Mariana. 2010. “Educación superior indígena en Brasil. Políticas gubernamentales y demandas indígenas: diálogos y tensiones”. Desacatos, 33: 67-84.

» PIÑA, Carlos. 1989. “Aproximaciones metodológicas al relato autobiográfico”. Revista Opciones, 16: 107-125.

» RAMOS, José Luis. 2010. “Las identidades étnica y nacional de maestros mixtecos (México), ¿Contradictorias o complementarias?”. Gazeta de Antropología, 26 (2). http://hdl. handle.net/10481/6769. (15 de septiembre de 2017).

» REA ÁNGELES, Patricia. 2011. “La educación superior como agente de transformación de las identidades genéricas entre los zapotecos en la ciudad de México”. Perfiles Educativos, 33: 226-238.

》 REBOLLEDO, Nicanor. 2009. Cultura, escolarización y etnografía. Los palikur en el Amazonas brasileño del Bajo Uaça. México: Universidad Iberoamericana.

»REMEDIOS, Alejandro Juan María y TAFOYA VARGAS, Cecilia. 2001. Maestros de educación indígena y revitalización cultural. http://tesiuami.izt.uam.mx/uam/aspuam/ver_texto.php?recno $=3885 \&$ documento=UAM3885.PDF. (01 de agosto de 2017).

» ROCKWELL, Elsie. 2009. La experiencia etnográfica. Historia y cultura en los procesos educativos. Buenos Aires: Paidós.

» ROCKWELL, Elsie. 2011: “Los niños en los intersticios de la cotidianeidad escolar ¿Resistencia, apropiación o subversión?”. En: G. Batallán y M. R. Neufeld (Coords.). Discusiones sobre infancia y adolescencia. Niños y jóvenes dentro y fuera de la escuela. Buenos Aires: Biblos. pp. 27-52.

» ROCKWELL, Elsie. 2013. “El trabajo docente hoy: nuevas huellas, bardas y veredas”. Congreso Nacional de Investigación Educativa. Conferencias Magistrales. México: Consejo Mexicano de Investigación Educativa.

» SALTALAMACCHIA, Homero, COLÓN, Homero y RODRÍGUEZ, Javier. 1983. “Historias de vida y movimientos sociales: propuestas para el uso de la técnica”. Revista Iztapalapa, 9: 321-336.

» SCHADEN, Ergon. 1998 [1954]. Aspectos fundamentales de la cultura guaraní. Asunción: Universidad Católica.

» SCOTT, Joan. 2010. “Género ¿Todavía una categoría útil para el análisis?”. Revista Diógenes 57(1): 7-14.

»SEGATO, Rita. 2011. "Género y colonialidad: en busca de claves de lectura y de un vocabulario estratégico descolonial”. En: K. Bidaseca y L. Vázquez (Comps.). Feminismos y poscolonialidad. Descolonizando el feminismo desde y en América Latina. Buenos Aires: Ediciones Godot. pp. 17-47. 
» SERRANO RUIZ, Javier. 1998. “El papel del maestro en la Educación Intercultural Bilingüe”. Revista Iberoamericana de Educación, 17: 91-102.

"SERRUDO, Adriana. 2010. "Indígenas en la escuela: representaciones y tensiones acerca de los docentes indígenas bilingües en Argentina". En: S. Hirsch y A. Serrudo (Comps.). La educación intercultural bilingüe en Argentina: identidades, lenguas y protagonistas. Buenos Aires: Noveduc. pp. 255-272.

» SUSNIK, Bratislava. 1983. Los aborígenes del Paraguay. Asunción: Museo Etnográfico Andrés Barbero.

"VALDEZ, María Cristina, RODRÍGUEZ DE ANCA, Alejandra, VILLARREAL, Jorgelina, ALVES, Ana. 2015. "Maestros mapuce en escuelas públicas insertas en lofce: historia, tensiones y desafíos para una educación intercultural". En: A. C Hecht y M. A Schmidt (Comps.). Maestros de la Educación Intercultural Bilingüe. Buenos Aires: Novedades Educativas. pp. 47-68.

" VÁZQUEZ GARCíA, Carolina. 2012. "Miradas de las mujeres ayuujk. Nuestra experiencia de vida comunitaria en la construcción del género". En: R. Hernández y A. Canessa (Eds.). Complementariedades y exclusiones en Mesoamérica y los Andes. Quito: Editorial Universitaria Abya-Yala. pp. 319-328.

»VILLARREAL, María Claudia y MARTíNEZ, María Eugenia. 2015. "Maestros indígenas en la provincia de Santa Fe". En: A. C. Hecht y M. A. Schmidt (Comps.). Maestros de la Educación Intercultural Bilingüe. Buenos Aires: Novedades Educativas. pp. 89-104.

"ZÚÑIGA, Marianela. 2017. Educación intercultural bilingüe, políticas educativas neoliberales y desigualdad: el caso de los docentes awajún de la región San Martín, Tesis de Maestría, Facultad Latinoamericana de Ciencias Sociales (FLACSO- Sede Argentina). 